Musées, Patrimoine et Culture scientifiques et techniques

175 | 2018

janvier-février 2018

\title{
Musée + Art-thérapie = Muséothérapie ?
}

\section{Mélissa Nauleau}

URL : http://journals.openedition.org/ocim/1896

DOI : 10.4000/ocim.1896

ISSN : 2108-646X

\section{Éditeur}

OCIM

Édition imprimée

Date de publication : 1 janvier 2018

Pagination : 16-21

ISSN : 0994-1908

Référence électronique

Mélissa Nauleau, « Musée + Art-thérapie = Muséothérapie? », La Lettre de l'OCIM [En ligne], 175 | 2018, mis en ligne le 01 janvier 2019, consulté le 22 avril 2019. URL : http://journals.openedition.org/ ocim/1896; DOl : 10.4000/ocim.1896

Ce document a été généré automatiquement le 22 avril 2019.

Tous droits réservés 


\title{
Musée + Art-thérapie = Muséothérapie?
}

\author{
Mélissa Nauleau
}

"Surmonter ses phobies", expérience menée au Bristol City Museum and Art Gallery.

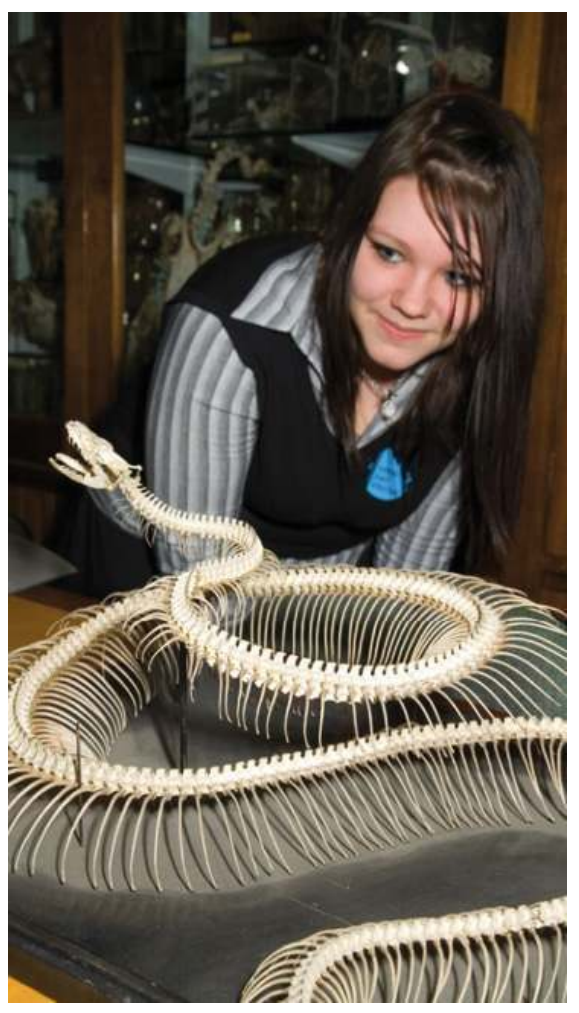

(c) DR

Le 4 novembre 2016, le musée des Beaux-Arts de Montréal inaugurait son nouveau Pavillon pour la Paix, abritant l'Atelier International d'éducation et d'art-thérapie Michel de la Chenelière. Nathalie Bondil, directrice de l'institution, accompagna les 
agrandissements du musée d'un Manifeste pour un musée humaniste défendant l'idée " qu'au XXI siècle la culture sera pour la santé ce que le sport a été au XXe siècle : l'expérience culturelle contribuera au mieux-être, comme la pratique du sport à notre forme physique" ${ }^{11}$. Le concept de musée thérapeutique, décrit par Nathalie Bondil sous l'appellation "muséothérapie", surprend et interroge par les bouleversements idéologiques, sociétaux et professionnels qu'il amorce. La fréquentation des musées, potentiellement bénéfique pour la santé, serait-elle susceptible d'être conseillée - pour ne pas dire prescrite - par les médecins?

2 Le concept de musée thérapeutique, ou muséothérapie, peut être défini comme suit : " Exploitation de l'environnement muséal et de ses collections à des fins de bien-être, de mieux-être ou d'amélioration de l'état de santé des participants."

\section{Art-thérapie et Arts in Health : aux origines de la muséothérapie}

3 L'avènement des pratiques muséales à vocation thérapeutique découle d'une succession et d'un enchevêtrement d'évènements ayant progressivement conduit à la reconnaissance des bienfaits de l'art sur la santé. Étroitement liée à la naissance de l'artthérapie, cette idée - d'abord philosophique, artistique puis médicale - selon laquelle la contemplation et la pratique des arts, tous confondus, favorisent le rétablissement des malades et le maintien d'un bon état de santé, ne pouvait se tenir plus longtemps à l'écart des musées.

Les premiers projets conduits en ce sens virent le jour en 1944, au Royaume-Uni, avec le concours du peintre Adrian Hill (1895-1977), reconnu comme l'un des fondateurs de l'artthérapie. Fort des succès rencontrés par les ateliers de peinture qu'il dispensait aux patients de sanatoriums au lendemain de la Seconde guerre mondiale, Adrian Hill convainquit la British Red Cross Society que le contact avec l'art améliorait le bien-être et favorisait la guérison des malades. Ensemble, ils créèrent le Red Cross Picture Library Scheme, un programme visant à constituer une bibliothèque de reproductions photographiques de chefs-d'œuvre de l'histoire de l'art, destinées à être exposées dans des hôpitaux britanniques, afin de soulager les maux des patients ${ }^{2}$. En l'espace de six années, plus de 4000 reproductions furent envoyées à près de deux cent hôpitaux. Le Red Cross Picture Library Scheme, tournant majeur dans la reconnaissance de l'art-thérapie, constitue un jalon des interventions muséales en milieu hospitalier telles que nous les connaissons aujourd'hui. Aux expositions organisées dans les espaces communs furent rapidement ajoutées des interventions de conférenciers. Présents une fois par semaine sur les lieux, les intervenants laissaient aux patients le choix d'exposer dans leur chambre une œuvre sélectionnée parmi les reproductions proposées, puis partaient de celle-ci pour lancer toutes sortes de conversations et débats. Par ce système de diffusion des œuvres et cette nouvelle forme de médiation culturelle - déjà conversationnelle exportés en milieu hospitalier, l'art-thérapie initiée par Hill annonçait les futurs développements de l'offre muséale à vocation thérapeutique et ses dispositifs de médiation spécifiques. 
Carte régionale des activités pour la santé et le bien-être dans les musées britanniques (Lackoi, K., Patsou, M. et Chatterjee, H. Museums for Health and Wellbeing. A Preliminary Report, National Alliance for Museums, Health and Wellbeing, 2016).

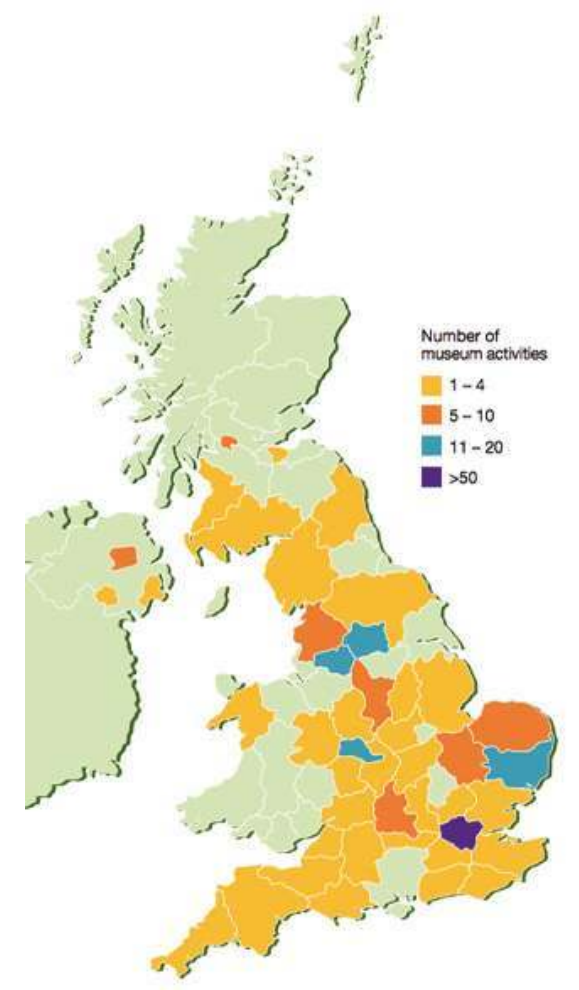

Les premiers développements de l'art-thérapie au Royaume-Uni exercèrent une influence majeure aux États-Unis et au Canada, engageant à leur tour les développements de la discipline et son intégration au sein des établissements de soins. En France, en revanche, son émergence fut étroitement rattachée à la sphère psychiatrique d'abord, artistique ensuite. Les médecins, puis les artistes, intellectuels et collectionneurs, s'approprièrent l'art-thérapie non pas sous l'angle des bienfaits qu'elle pouvait conférer aux patients, mais sous le prisme de la qualité artistique que pouvaient revêtir les œuvres de certains "patients-artistes", rapidement rattachés, malgré eux, à l'art brut ${ }^{3}$. Demeurant prisonnière de l'étiquette d'un art qualifié de "psychopathologique", la discipline fut freinée dans son émancipation, de même que furent écartés ses bénéfices thérapeutiques. Encore aujourd'hui, alors que le Royaume-Uni, les États-Unis et le Canada accordent le titre d'art-thérapeute uniquement aux personnes titulaires d'un diplôme spécialisé, la France peine à harmoniser la formation - initiale comme continue - de l'art-thérapie. Ce retard dans la reconnaissance et dans la professionnalisation entraine dès lors des difficultés de définition, mais également de légitimité pour la discipline, déjà largement pratiquée et reconnue dans les pays anglo-saxons.

Par le biais de ces déploiements de l'art-thérapie, les pays anglo-saxons aboutirent progressivement à la définition d'un champ de recherche et de pratiques culturelles spécifique : les "Arts in Health" (les arts en santé) ou "Arts in Healthcare" (les arts dans les soins de santé). Apparu dans les années 1990 au Royaume-Uni, ce champ particulier témoigne de la capacité des professionnels anglo-saxons à faire rencontrer arts et santé, en théorie comme en pratique, dans la médecine comme dans les sciences humaines. Dans cette logique, les rapports entre les arts et la santé s'affirmèrent au point de constituer de 
nouveaux axes stratégiques des politiques de santé publique. Aujourd'hui, le RoyaumeUni, les États-Unis, le Canada et d'autres pays anglo-saxons possèdent des réseaux nationaux, voire gouvernementaux, dont la mission consiste à développer et soutenir la recherche ainsi que l'usage des arts en santé. Persuadés et persuadant que les arts jouent un rôle majeur dans l'amélioration du bien-être et de la santé des populations, les politiques, professionnels et chercheurs anglo-saxons ne cessent de resserrer les liens entre les institutions culturelles et les institutions de santé.

7 Le Royaume-Uni fut le premier pays à engager puis défendre le ralliement des musées à ce phénomène. Par l'alliance de la muséologie, de la médecine et des politiques de santé publique, une nouvelle spécialité de la recherche et des pratiques muséales fit ainsi son apparition : "Museums in Health"4. L'émergence de ce champ spécifique aux musées témoigne de la considération progressive de cette mutation discrètement engagée depuis une dizaine d'années et marque, par ailleurs, le passage d'une politique muséale à vocation non plus uniquement sociale, mais également thérapeutique.

\section{Les musées anglo-saxons : alliés de la santé publique}

8 Le concept de musée thérapeutique s'est affirmé au Royaume-Uni dans les années 2000, bien que l'idée en elle-même, difficile à dater de manière précise, lui soit largement antérieure. La publication d'un dossier spécialement consacré à ce sujet dans la revue Museum Practice en 2009 figea l'expression "therapeutic museums" ${ }^{1}$ : une terminologie surprenante et marginale à l'époque, reprise et développée dans de nombreux pays anglosaxons aujourd'hui.

C'est avec la réforme du système de santé britannique en 2012 que les politiques anglosaxonnes commencèrent officiellement à entraîner les musées dans la lutte contre les inégalités en santé et pour l'amélioration du bien-être des populations ${ }^{6}$. Dans un contexte où l'inclusion sociale menée par le biais de l'institution muséale était déjà promue et soutenue par le gouvernement, les musées britanniques devaient s'attacher à trouver de nouvelles raisons permettant de justifier le maintien de leurs financements. Alors que de nombreuses publications scientifiques alarmaient sur le fait que les pays occidentaux et industrialisés connaissent des défis socio-économiques majeurs auxquels ils doivent faire face pour la première fois (dysfonctionnements dans les systèmes de santé, accroissement des maladies liées au vieillissement et aux modes de vie malsains, inégalités croissantes dans les accès aux soins, répartition inégale des richesses), le gouvernement britannique fut forcé de constater qu'il était désormais indispensable de mettre en place des politiques de santé fondées sur la prévention des maladies et sur l'amélioration du bienêtre général des populations.

C'est ainsi que le Royaume-Uni d'abord, les États-Unis et le Canada ensuite, encouragèrent les développements d'une vision holistique de la santé publique. Cette approche, liée à la reconnaissance de l'influence directe des facteurs sociaux et environnementaux sur la santé, fit progressivement des musées les alliés évidents de nouvelles politiques de santé. Dans ce contexte d'interrogations sanitaires, économiques, politiques et sociales, les musées, alors considérés comme de possibles vecteurs de diffusion des savoirs et des pratiques en santé, s'engagèrent dans une nouvelle voie thérapeutique. 
Kit de mesure du bien-être mis au point par l'University College London et utilisé par les musées partenaires du programme.

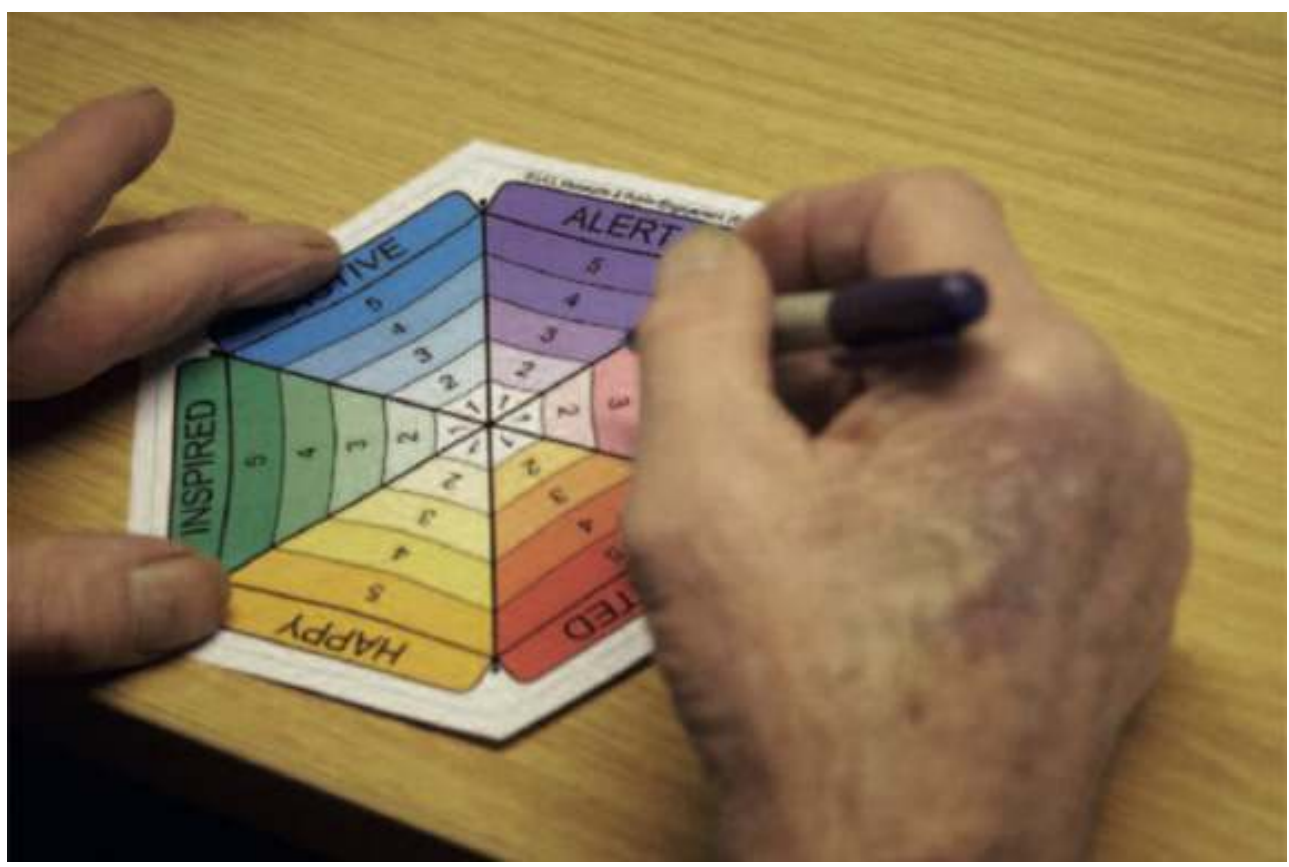

(c) DR

11 En 2016, la National Alliance for Museums, Health and Wellbeing publia une étude consacrée à ce phénomène, alors étendu sur l'ensemble du Royaume-Uni ${ }^{7}$. L'Alliance dénombra au total 603 projets, programmes ou évènements, établis sur du court ou du long terme, rodés ou en cours de conception, émanant de 261 musées britanniques. L'ensemble des projets répertoriés dans le cadre de cette étude permettait de souligner :

12 - la diversité des publics ciblés par ces pratiques : grand public (adultes comme enfants, individuel comme familial), personnes âgées, personnes atteintes de démences, de handicaps ou de troubles psychiques, personnes hospitalisées ou issues de classes sociales défavorisées, sans-abris, personnes sans-emplois... ;

13 - la capacité des musées à innover, notamment dans la création de nouveaux dispositifs de médiation : visites, ateliers, mallettes ou applications spécifiquement conçus pour de nouveaux publics ;

14 - la possibilité pour les institutions de réadapter des offres existantes selon des objectifs d'amélioration du bien-être : intégration de marches de santé ou d'exercices de yoga dans les parcours de visite, activités participatives et conversationnelles axées sur la réminiscence ou l'expression de soi, ateliers de pratique artistique réorientés...

Cette multitude de projets recensés, couplée à la diversité d'activités et de cibles, témoigne cependant de la difficulté à distinguer bien-être et thérapie. À quel moment peut-on considérer qu'un musée joue véritablement un rôle thérapeutique?

Une recherche-pilote menée entre 2013 et 2014 dans l'East Midlands, une région du Royaume-Uni, illustre la diversité des applications qu'il est possible d'envisager et de mettre en place dans cette perspective thérapeutique ${ }^{8}$. Cinq musées de la région sélectionnée en raison de sa démographie restreinte et pour ses caractéristiques rurales collaborèrent avec des structures du secteur social et de la santé afin de rendre compte 
du rôle que les musées, toutes échelles et collections confondues, peuvent jouer en matière de santé publique, cela y compris dans les régions les plus défavorisées du pays. Trois grands projets virent ainsi le jour : "Encountering the Unexpected" (consacré au vieillissement et à l'isolement des personnes âgées), "Live today, Think tomorrow" (pour la prévention du tabagisme dans les établissements scolaires) et "Body, Mind, and Spirit" (création d'une plate-forme en ligne pour les enfants hospitalisés). Au total, 93 témoignages de participants furent recueillis. En s'appuyant sur un kit de mesure du bien-être mis au point par l'University College London, ces activités révélèrent l'augmentation significative de différentes émotions liées au bien-être : joie, enthousiasme, activité, inspiration et vivacité. Cette augmentation s'avéra nettement plus élevée que dans d'autres régions plus urbanisées où des expériences similaires avaient également pu être menées. La recherche soulignait ainsi toute l'importance et l'intérêt d'investir les musées régionaux, notamment les plus modestes. De fait, le succès rencontré par ces programmes, ainsi que les résultats positifs issus de leur évaluation, encouragèrent les institutions à maintenir leurs partenariats, voire à étendre leurs projets.

D'autres expériences britanniques semblent confirmer le potentiel thérapeutique des musées et de leurs collections. Certains projets, spécifiquement conçus pour lutter contre une pathologie ou un trouble, témoignent de la possibilité des musées d'investir totalement leurs collections dans une visée de soin : usage de techniques médicales visant à stimuler et raviver la mémoire de visiteurs atteints de la maladie d'Alzheimer ; soulagement de troubles liés à des traumatismes, notamment chez les anciens combattants ; exploitation de spécimens naturalisés en soutien à des traitements contre diverses phobies... De surcroît, les musées étant particulièrement soucieux de (dé)montrer leurs potentiels bienfaits thérapeutiques, ce type d'activités se trouve soumis à des procédures d'évaluation, comme de suivi. 
Rencontres inhabituelles au Crich Tramway Village \& Museum

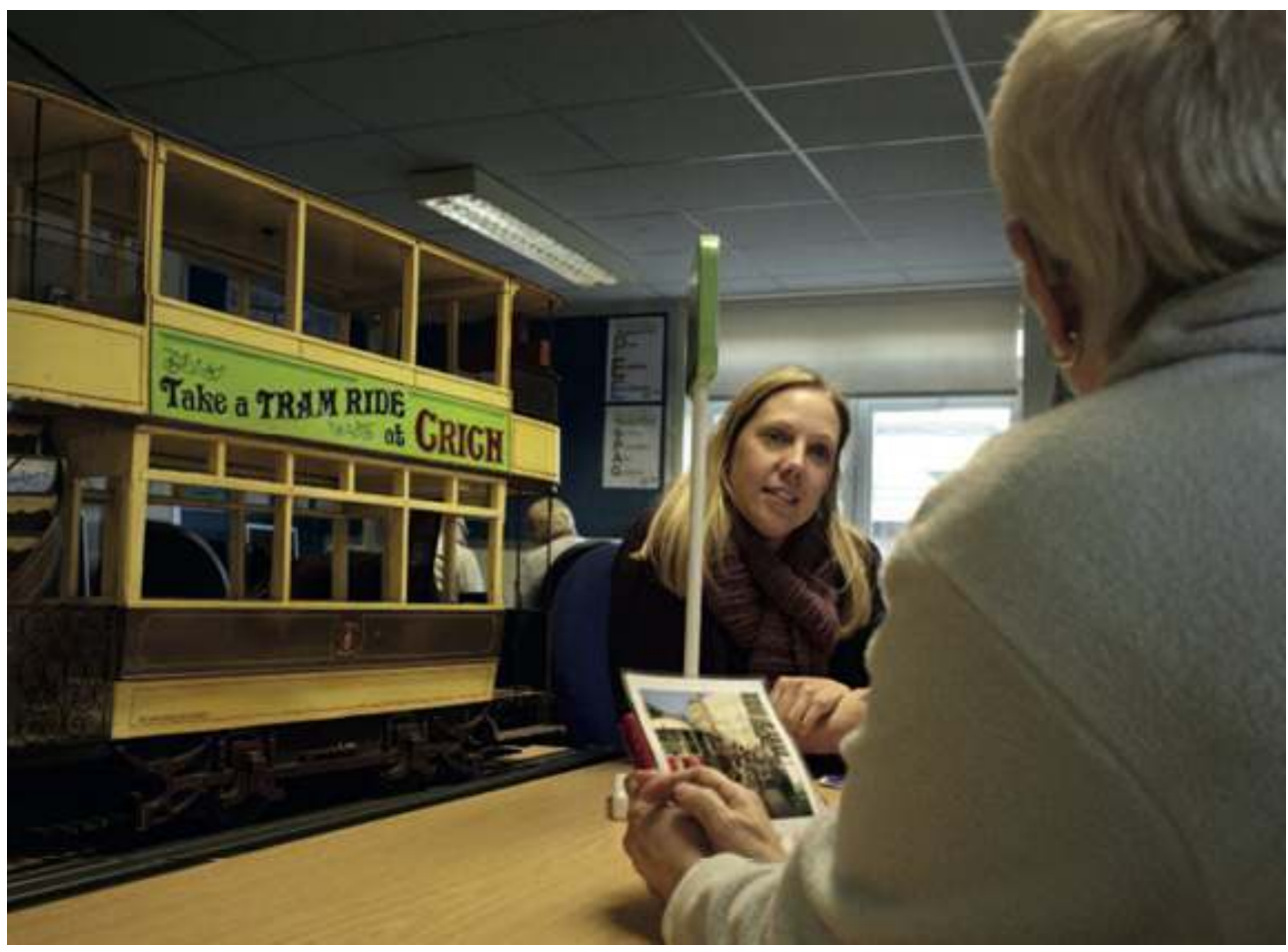

(C) DR

Le projet "Meet Me" a été mené au MoMA à New York en 2015.

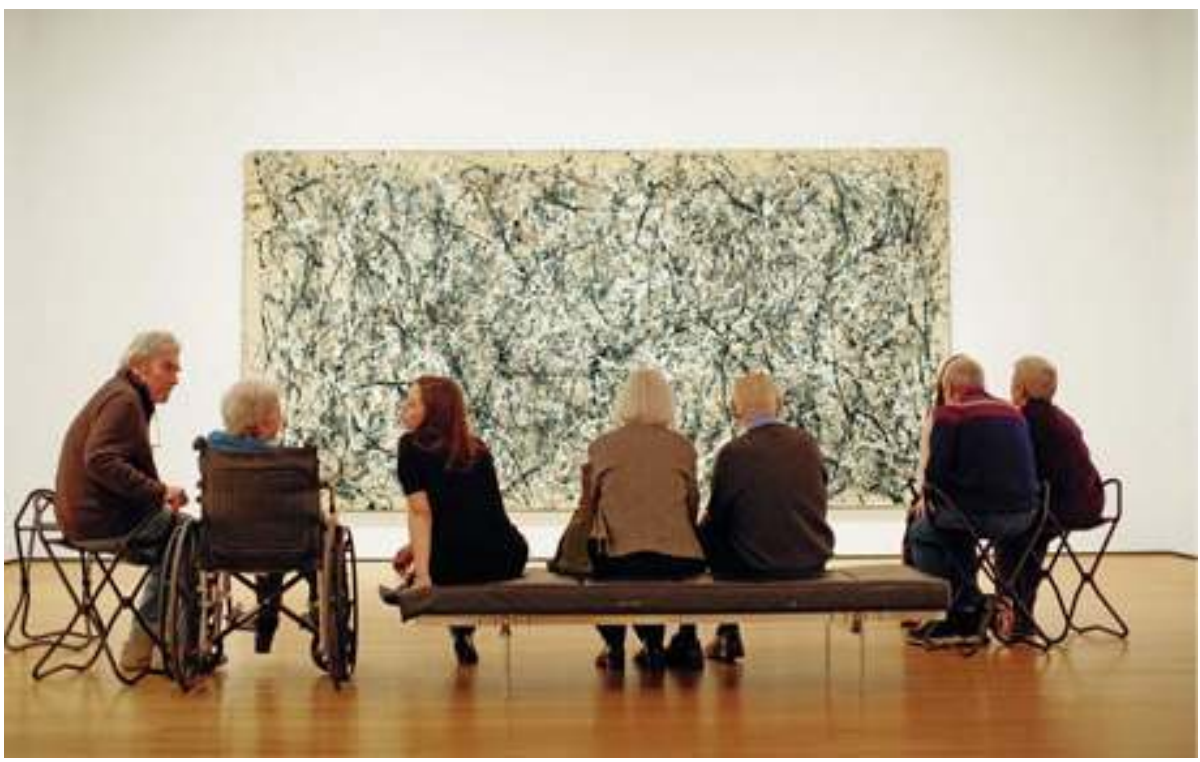

(c) MoMA/Jason Brownrigg

\section{L'influence internationale des musées nord-américains}

Dans la lignée de ces premières expériences britanniques encourageantes, les musées nord-américains développèrent à leur tour des projets à visée thérapeutique. C'est ainsi 
que deux évènements majeurs marquèrent le basculement officiel et international des institutions dans la muséothérapie.

Le premier fut le lancement, en 2014, du programme "Meet Me" par le MoMA de New York. Bien que l'institution n'employa jamais le terme "thérapie" pour désigner ses activités, ce premier projet muséal à destination des personnes atteintes de la maladie d'Alzheimer affirmait, pour la première fois et à l'adresse des musées du monde entier, que tout établissement serait désormais en mesure de soutenir la lutte contre la maladie, et notamment de ralentir certains de ses effets (troubles du comportement, de l'humeur, dépression...), recherche médicale à l'appui.

Deux années plus tard, l'inauguration de l'Atelier International d'éducation et d'artthérapie du musée des Beaux-Arts de Montréal (MBAM) marqua un second tournant dans l'établissement de ce phénomène. L'intégration d'espaces spécifiquement consacrés à l'art-thérapie en plein cœur du musée assurait non seulement la visibilité de cette nouvelle offre, mais aussi la transmission des pratiques qui, jusqu'ici relayées par des publications scientifiques anglo-saxonnes, semblaient tenues à l'écart des professionnels français.

21 Aujourd'hui, le musée des Beaux-Arts de Montréal se positionne sur les devants de la scène de la muséothérapie, avec la ferme volonté de faire reconnaître les bienfaits de la culture pour la santé, et plus particulièrement ceux du musée. Sur son site Internet, l'institution affiche l'art-thérapie aux côtés de l'éducation. Le musée développe en effet une multitude de programmes spécifiquement conçus pour répondre aux besoins médicaux, psychologiques ou sociaux de ses participants : démences, autisme, troubles de l'alimentation, bégaiement, maladies cardio-vasculaires, problématiques liées au suicide et à la radicalisation... La liste, non exhaustive, place le MBAM en tête des institutions muséales ayant à cœur de devenir l'allié indispensable des politiques publiques, sociales comme de santé. En s'imposant comme un véritable "musée-laboratoire", les frontières qui pouvaient encore séparer sphères médicale et muséale sont désormais totalement abolies. Pour preuve supplémentaire, le musée créa en janvier 2017 son comité consultatif "Art et Santé". Conscient des retards du Canada en matière de recherche sur l'utilisation des arts en santé (le Royaume-Uni et les États-Unis se maintenant en tête dans ce domaine), le comité du MBAM prévoit d'accompagner le développement de ses pratiques thérapeutiques d'une démonstration et d'un suivi scientifiques à la hauteur des mutations muséales en train de s'opérer. Dirigé par un scientifique renommé et composé d'experts issus des milieux de la santé, de l'art-thérapie, de la recherche et des arts, ainsi que de représentants de la philanthropie montréalaise, ce comité incarne l'intégration idéologique comme organique - de la médecine au sein du musée.

Quant aux États-Unis et selon l'American Alliance of Museums, chaque État fédéré possède au moins un programme muséal lié à une problématique de santé publique ${ }^{9}$. En tête du classement des publics ciblés figurent, largement majoritaires, les participants atteints de la maladie d'Alzheimer et de démences similaires, suivis des personnes souffrant d'autisme. L'ensemble du pays, convaincu des bienfaits des arts sur la santé, a ainsi largement intégré les musées dans cette dynamique thérapeutique. 


\section{Réticences dans les musées français}

23

La question de l'intégration de l'art-thérapie - et plus largement de la thérapeutique - au sein des musées français est survenue avec l'avènement de l'offre à destination des personnes atteintes de la maladie d'Alzheimer. Influencés par l'initiative du MoMA, de nombreux professionnels se sont à leur tour lancés dans la conception de programmes destinés à ce nouveau public. Immédiatement, l'art-thérapie fut convoquée et questionnée. La configuration ralliant public malade, exploitation des collections et ateliers de pratique artistique permettait en effet de penser que les musées s'appuyaient désormais sur la discipline. Et, presque inévitablement, cela conduisait à désigner ces activités comme étant de l'art-thérapie. Mais, aussi rapidement qu'il était possible d'effectuer ce raccourci terminologique, l'art-thérapie fut d'emblée réfutée, les professionnels de musées exprimant en chœur que le rôle thérapeutique ne serait pas endossé par l'institution.

En 2014, à Dreux, l'exposition Premier pas 2014 présentait des œuvres de patients de l'hôpital Victor Jousselin réalisées au musée d'Art et d'Histoire de la ville (extrait de l'article d'Axelle Marin, Quand le musée soigne, publié dans La Lettre de l'Ocim, $\mathrm{n}^{\circ} 157,2015$, pp. 12-17).

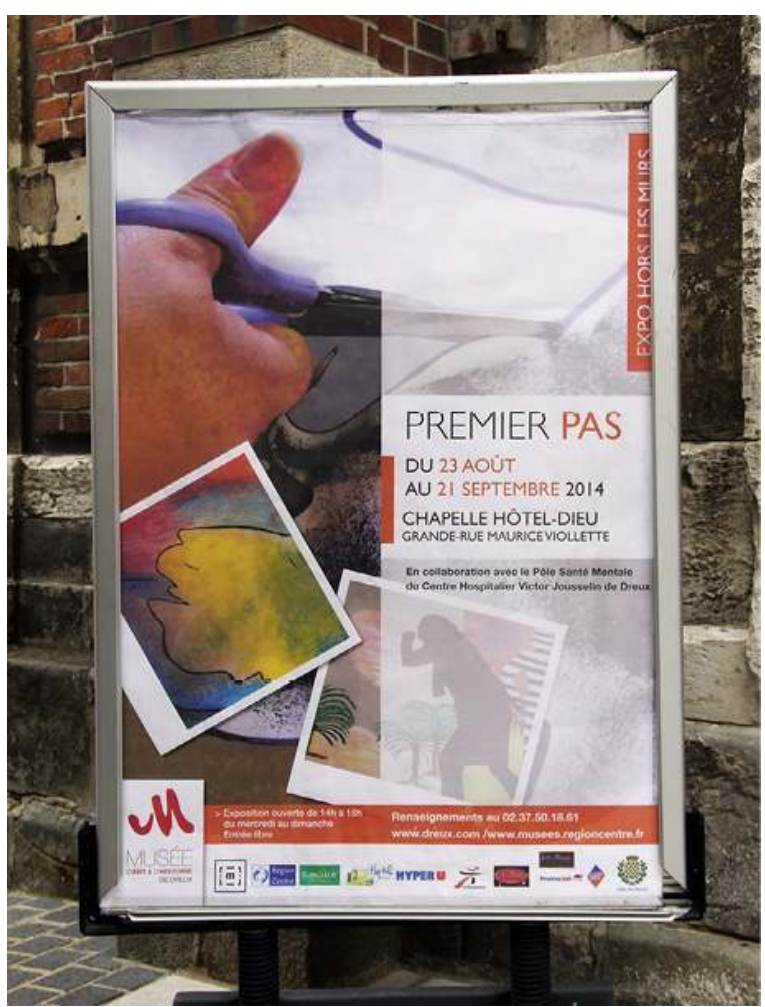

(c) Musée d'Art et d'Histoire, Ville de Dreux/AR

Il est pourtant aisé de remarquer que les projets français possèdent bon nombre de points communs avec les programmes britanniques, américains et canadiens. Eux aussi parsèment le paysage muséal et témoignent d'une certaine pratique thérapeutique menée par les institutions. Le programme "Au rythme du souvenir" du musée de la Musique de Paris rappelle, à bien des égards, "Meet Me" du MoMA, dont il s'est officiellement inspiré. Le muséum-aquarium de Nancy quant à lui, après s'être particulièrement concentré sur 
les personnes âgées atteintes des maladies d'Alzheimer ou de Parkinson, s'est considérablement rapproché des expériences britanniques en proposant des activités destinées aux seniors afin de lutter contre les troubles liés au vieillissement. Le Palais des Beaux-Arts de Lille illustre, de son côté, la capacité des musées à créer un environnement et des pratiques favorisant le bien-être des enfants autistes. De même, le CAPC musée d'Art contemporain de Bordeaux témoigne des avantages à faire collaborer les équipes des musées et des hôpitaux avec des artistes et des art-thérapeutes pour soulager les maux des enfants hospitalisés. Dans cette même ville, le musée d'Aquitaine aborde au travers de ses collections les problématiques du corps pour venir en aide aux adolescents souffrant de dépression, d'anorexie ou de boulimie. Le musée des Beaux-Arts de Caen intervient quant à lui auprès de patients d'un service d'addictologie afin de les soutenir dans leurs traitements. Ces quelques exemples sont loin d'être exhaustifs. On dénombre sur le territoire quelques centaines de programmes destinés à des publics spécifiques, notamment malades et menés dans cette même dynamique de partenariats auprès de structures spécialisées (hôpitaux, maisons de retraite, centres d'accueil, associations...). Et, comme pour les musées anglo-saxons, les typologies d'activités qu'il est possible de recenser sont sensiblement similaires : visites et ateliers adaptés, mallettes accompagnant les activités menées hors-les-murs, médiation conversationnelle... Aussi, lorsque que l'on questionne les professionnels en charge de ce type de projets quant aux objectifs fixés et aux résultats obtenus, tous font part d'observations positives identiques : succès croissant constaté auprès des participants, création de lien social, amélioration du bien-être ou encore réduction de certains troubles liés aux pathologies dont les participants sont atteints.

Malgré ces similitudes, la muséothérapie en France n'est pas (encore) à l'ordre du jour. Si les programmes français et anglo-saxons sont bien comparables, les premiers ont été mis en place dans le cadre de la mission de service public à laquelle les institutions se doivent de répondre. Il s'agit donc de rendre les collections accessibles à tous et, si rôle à jouer il y a, il n'est que d'ordre social. Bien que les collaborations entre les professionnels des musées, de la santé et du champ social soient aujourd'hui quasiment systématiques - et bénéfiques - les musées défendent la nécessité de maintenir une frontière nette entre ces différents secteurs. Et les objectifs thérapeutiques sont ainsi exclusivement portés par les professionnels de la santé, de même que l'évaluation des projets, extrêmement lacunaire, pour ne pas dire inexistante.

Il faut dire, qu'en France, la convention "Culture et Santé" maintient les offres muséales en lien avec la santé dans une vocation de démocratisation culturelle. L'influence des premières versions de cette convention interministérielle persiste, définissant les projets liés à cette politique comme des occasions d'établir un contact entre les publics issus du champ de la santé et les équipements culturels. L'accessibilité et la démocratisation demeurent ainsi les principaux objectifs, substantiellement indétrônables, de ce type d'activités. 
Le muséum-aquarium de Nancy a décidé de mettre en place des activités spécifiques à destination d'un public de personnes âgées atteintes de la maladie d'Alzheimer : visite-test dans la galerie de zoologie avec un groupe de personnes âgées d'un Ehpad (extrait de l'article de Caroline Grandjean et Marlène Prost, Des seniors au muséum : une nébuleuse de projets, publié dans La Lettre de l'Ocim , n¹49, 2013, pp. 5-10).

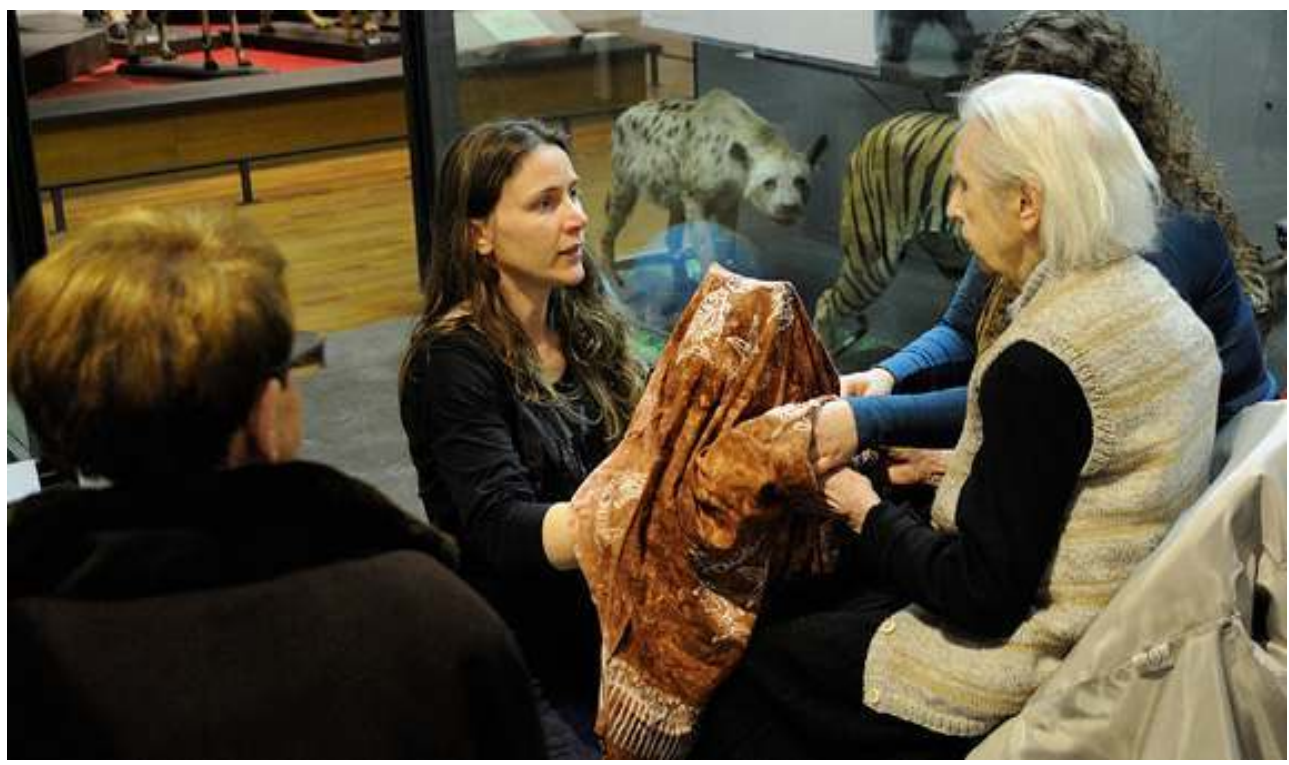

(c) Muséum-Aquarium de Nancy

Par ailleurs, l'art-thérapie est une discipline encore mal définie et donc relativement incomprise, mais également peu professionnalisée et par conséquent non reconnue. Dans ce contexte, les collaborations entre les médiateurs culturels et les art-thérapeutes sont particulièrement complexes et révèlent, bien souvent, des incompréhensions et des concurrences entre ces professionnels. La définition du rôle de chacun semble délicate et ralentit considérablement l'établissement de projets dans lesquels un réel partage des expertises et des savoir-faire pourrait s'instaurer.

Enfin, les lacunes dans la recherche (fondamentale comme appliquée), ainsi que l'insuffisante évaluation des programmes muséaux, freinent les initiatives possibles à mener dans ce champ d'expérimentation, peut-être encore trop original, sinon complexe par la pluridisciplinarité qu'il convoque.

Pourtant, aujourd'hui et notamment avec le concours du musée des Beaux-Arts de Montréal, une indubitable redéfinition du rôle des musées s'opère. D'autant plus que les professionnels des musées français, jusqu'ici relativement peu informés de ces expériences anglo-saxonnes, accèdent désormais à des ressources francophones, largement diffusées par l'institution montréalaise. Et, s'il s'avérait que les développements de ces pratiques muséales thérapeutiques continuaient de suivre le déroulement historique et géographique exposé préalablement : se pourrait-il que les musées français y deviennent, finalement, favorables? 


\section{BIBLIOGRAPHIE}

Camic, P. et Chatterjee, H. Museums and art galleries as partners for public health interventions, Perspectives in Public Health, vol.133, n¹, 2013, pp. 66-71.

Marin, A. Quand le musée soigne, La Lettre de l'Ocim, n¹57, 2015, pp. 12-17.

Tariant, E. Et si les musées soignaient ! Le Journal des Arts, n437, 2015.

Vandeninden, E. Devenir art-thérapeute, SociologieS (en ligne), 2014.

Waller, D. Becoming a Profession : The History of Art Therapy in Britain 1940-82. London : Routledge, « Psychologie Revivals », 1991, 304 p.

\section{NOTES}

1. Bondil, N. (dir.) Pavillon pour la paix Michal et Renata Hornstein : Art International et éducation, Atelier international d'éducation et d'art-thérapie Michel de la Chenelière. Montréal : Éditions du Musée des Beaux-Arts de Montréal, 2016, p. 22.

2. Hogan, S. Healing Arts : The History of the Art Therapy. London : Jessica Kingsley Publishers, 2001.

3. Dubois, A.-M. Art-Thérapie : Principes, méthodes et outils pratiques. Paris : Édition Elsevier Masson, 2013, pp. 6-8.

4. Chatterjee H. et Noble G. Museums, Health and Well-Being. Farnham: Ashgate, 2013, p. 2.

5. Therapeutic museums, Museum Practice, winter 2009, pp. 39-55.

6. Chatterjee, H. et Noble, G. Museums, Health and Well-Being. Farnham: Ashgate, 2013.

7. Lackoi, K., Patsou, M. et Chatterjee, H. Museums for Health and Wellbeing. A Preliminary Report. National Alliance for Museums, Health and Wellbeing, 2016. Disponible sur : https:// museumsandwellbeingalliance.files.wordpress.com/2015/07/museums-for-health-andwellbeing.pdf

8. Dodd, J. et Jones, C. Mind, body, spirit : how museums impact health and wellbeing. Leicester : School of Museum Studies and University of Leicester, juin 2014. Disponible en ligne sur hwww2.le.ac.uk/departments/museumstudies/rcmg/publications/mind-body-spirit-report

9. American Alliance of Museums. Museums On Call : How Museums Are Adressing, 2013. Disponible sur : www.aam-us.org/docs/default-source/advocacy/museums-on-call.pdf?sfvrsn=8

\section{RÉSUMÉS}

Le musée peut-il contribuer à soulager les maux du corps et de l'esprit ? Face à cette interrogation, l'auteur se penche sur le concept de muséothérapie, décrit les principes et expériences qui ont participé à sa naissance et à son développement dans les pays anglo-saxons. 
Elle analyse par ailleurs les difficultés rencontrées en France pour mettre en place des pratiques muséales à vocation thérapeutique.

INDEX

Mots-clés : musée, art-thérapie, santé

\section{AUTEUR}

\section{MÉLISSA NAULEAU}

muséologue, diplômée du Master de recherche en muséologie de l'École du Louvre, spécialité politique des publics

melissa.nauleau@gmail.com 